\title{
An uncooled VGA-IRFPA with novel readout architecture
}

\author{
D. Würfel, M. Ruß, R. Lerch, D. Weiler, P. Yang, and H. Vogt \\ Fraunhofer Institute for Microelectronic Circuits and Systems, Duisburg, Germany
}

\begin{abstract}
An uncooled VGA Infrared Focal Plane Array (IRFPA) based on microbolometers with a pixel pitch of $25 \mu \mathrm{m}$ for thermal imaging applications is presented. The IRFPA has a 16-bit digital video data output at a frame rate of $30 \mathrm{~Hz}$. Thousands of Analog to Digital Converters (ADCs) are located under the microbolometer array. One ADC consists of a Sigma-Delta-Modulator (SDM) of 2nd order and a decimation filter. It is multiplexed for a certain amount of microbolometers arranged in a so called "cluster". In the 1st stage of the SDM the microbolometer current is integrated time-continuously. The feedback is applied using a switchable current source. First measurements of Noise Equivalent Temperature Difference (NETD) as a key parameter for IRFPAs will be presented.
\end{abstract}

\section{Introduction}

Low-cost thermal imaging is dominated by uncooled infrared focal plane arrays (IRFPAs) using microbolometers based on vanadium oxide (Blackwell et al., 2008) or amorphous silicon (Durand et al., 2009) as the sensing material. According to Plank's law, each body with a temperature above absolute zero point emits electro-magnetic radiation. The wavelength and intensity depends on the temperature of the body. For example, a body at a temperature of $300 \mathrm{~K}$ has its maximum emittance at a wavelength of approx. $10 \mu \mathrm{m}$. FIR imager uses the IR-radiation of a warm body in the wavelength range between $8 . .14 \mu \mathrm{m}$. The FIR-sensitive element is the microbolometer which changes its resistance by absorbing the radiation of a warm body. Uncooled IRFPAs operate at ambient temperature and benefit from the abdication of a Stirling cooler in terms of low-cost, low power dissipation,

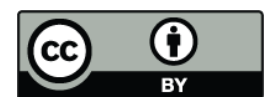

Correspondence to: D. Würfel (daniel.wuerfel@ims.fraunhofer.de)

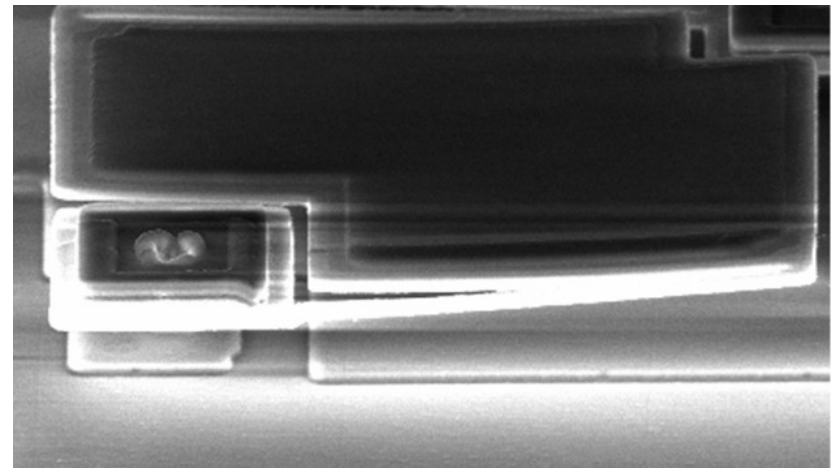

Fig. 1. SEM-photo of a microbolometer constructed at Fraunhofer IMS.

low weight, and reduced volume. Typical applications for IRFPAs are thermal imaging and pedestrian detection for automotive driving assistance systems, fire fighting, biological imagery, or military applications like target recognition.

\section{The microbolometer}

The microbolometer is fabricated by post-processing on CMOS wafers in Fraunhofer IMS Microsystem Lab. The realization of a microbolometer is shown in Fig. 1. A micromachined membrane consisting of amorphous silicon is suspended by two via stacks of metal from the CMOS substrate. The membrane forms with two other layers a good interferometric structure for radiation absorption. On top of the membrane an antireflection layer with a sheet resistance of $377 \Omega / \mathrm{sq}$ is deposited. The bottom structure consists of a nearly perfect reflecting metal layer (Ruß et al., 2007). To increase the thermal resistance the membrane is fixed by two small legs. The distance between membrane and reflection metal reaches an optimum for one quarter of the radiation

Published by Copernicus Publications on behalf of the URSI Landesausschuss in der Bundesrepublik Deutschland e.V. 


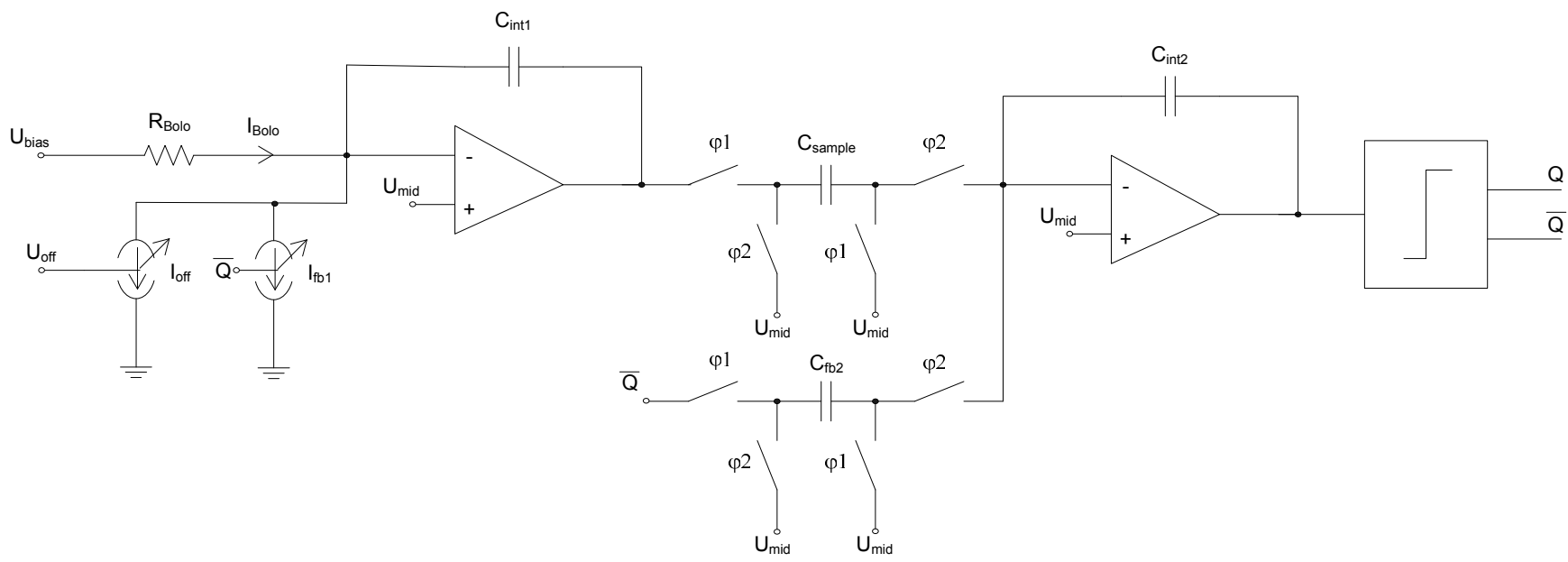

Fig. 2. Schematic of used 2nd Order SDM.

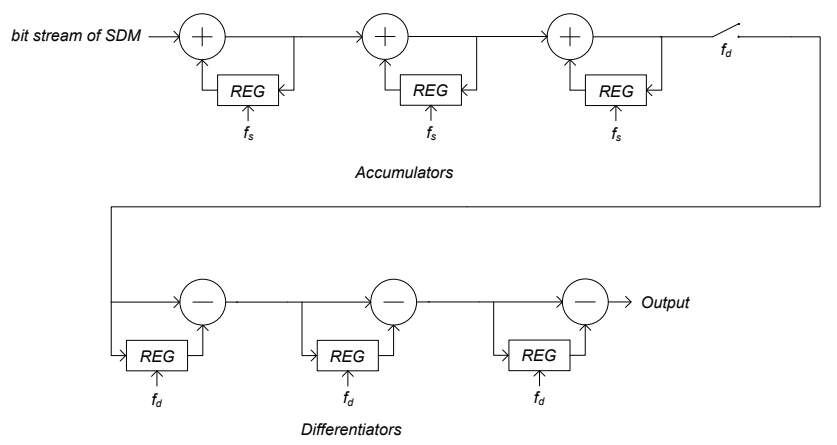

Fig. 3. Model of a 3rd Order SINC-Filter.

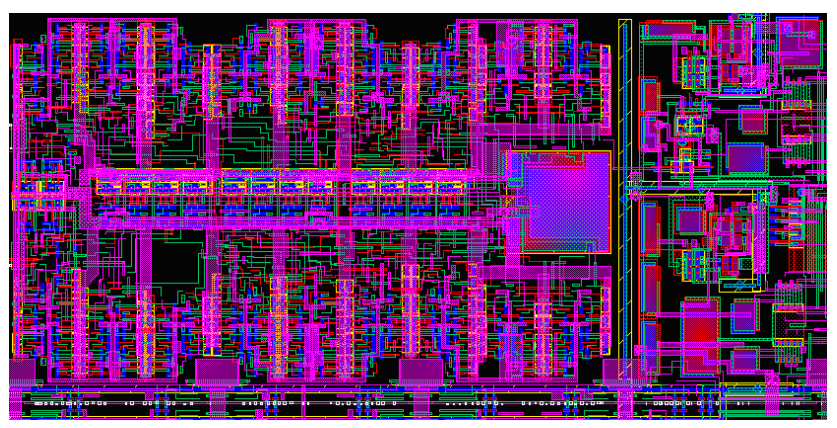

Fig. 4. Layout of a cluster (some metal layers are omitted for clarity).

wavelength. To reduce thermal losses by gas conduction a vacuum package is required. The microbolometer converts the infrared radiation into heat energy and this induces a temperature rise resulting in a change of the electrical resistance. The microbolometers are arranged in an array with a pixel pitch of $25 \mu \mathrm{m}$.

\section{The novel readout architecture}

The novel readout architecture depicted in Fig. 2 bases on a 2nd order SDM (Weiler et al., 2010). A SDM has the advantage of reaching high resolutions very easily. The price is sampling speed, because a SDM uses so called "oversampling". Quantisation noise is formed and moved out of the base band to higher frequencies. The output signal of a SDM is a digital bit stream which is fed into a decimation filter. This filter is a low-pass and suppresses the quantisation noise. The sampling speed is reduced while increasing the length of the output word. The SDM together with its decimation filter forms the ADC.

The for readout selected microbolometer is integrated directly into the SDM by closing the corresponding selectswitch. Reference $U_{\text {bias }}$ is a constant voltage. The microbolometer current consisting of an offset part and a signal part is integrated time-continuously. The feedback is applied by a switchable current source. A controllable offset current source is also included to compensate the offset part of the microbolometer current. The 2nd integrated stage works time-discretely. Because of the multiplexing of the readout circuit for 32 microbolometers a decimation filter with an finite settling time is necessary. So a 3rd order SINC-Filter was chosen for this function. Figure 3 shows a corresponding scheme based on (Norsworthy, 1997). It consists of 3 cascaded accumulators which are running at the sampling frequency $f_{s}$. Then there is a undersampling stage with frequency $f_{d}$. It follow 3 differentiators which are running at frequency $f_{d}$. The amount of 32 microbolometers which are read out by one ADC (consisting of the SDM and the decimation filter) is the result of the minimal area where one ADC can be placed. The ADC is arranged in a cluster consisting of 4 times 8 microbolometer areas. A layout of a cluster is depicted in Fig. 4. Three quarters of the area to the left are occupied by the digital SINC-Filter. The SDM is placed in 


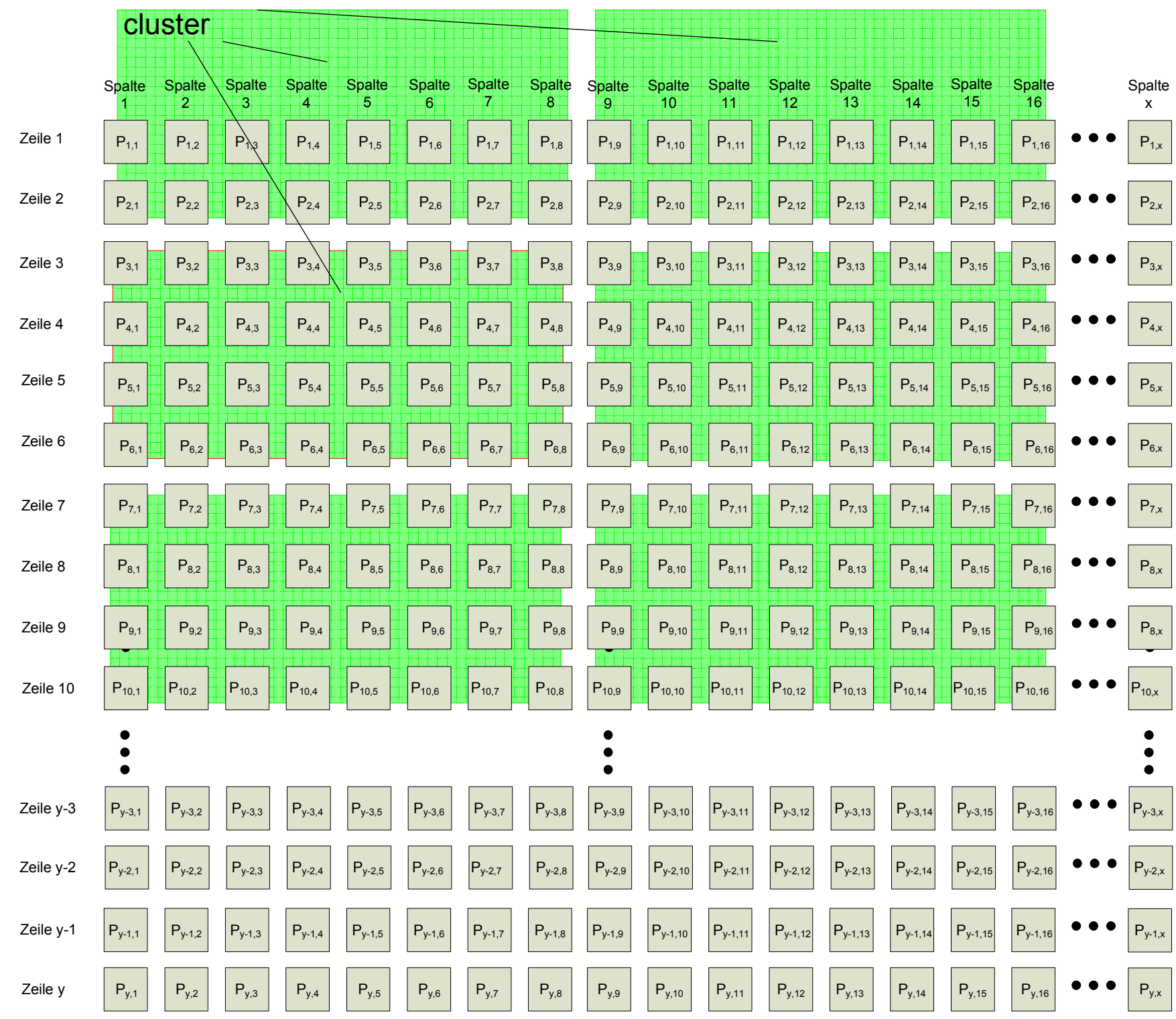

Fig. 5. Distribution of the clusters beneath the microbolometer array.

the right quarter. Beneath the whole microbolometer array approximately 10000 ADCs are positioned. In Fig 5 in a cut-out the positions of some clusters beneath the array are shown. The digital output from the ADCs is multiplexed to a 16 digital video output interface. The frame rate is $30 \mathrm{~Hz}$. The IRFPA has been fabricated in a $0.35 \mu \mathrm{m}$ CMOS technology and bonded on a PCB-board (Fig. 6).

\section{First measurement results}

For first measurements the chip with microbolometers of the 1st generation was placed in a vacuum chamber with an FIR transparent window. A measured NETD histogram is shown in Fig. 7. The mean value of the NETD is approximately $150 \mathrm{mK}$. The value is higher than the design value but it has to be considered that it is the 1st generation of microbolometers and the microbolometer resistance failed the goal resistance extensively. But for all the VGA-IRFPA works promisingly. A picture of a hand has been taken (Fig. 8). It has to be remarked that beside an offset correction no further correction or filter is applied to the picture.

\section{Conclusions}

A VGA-IRFPA with a novel readout architecture has been presented. It is based on massively parallel use of 


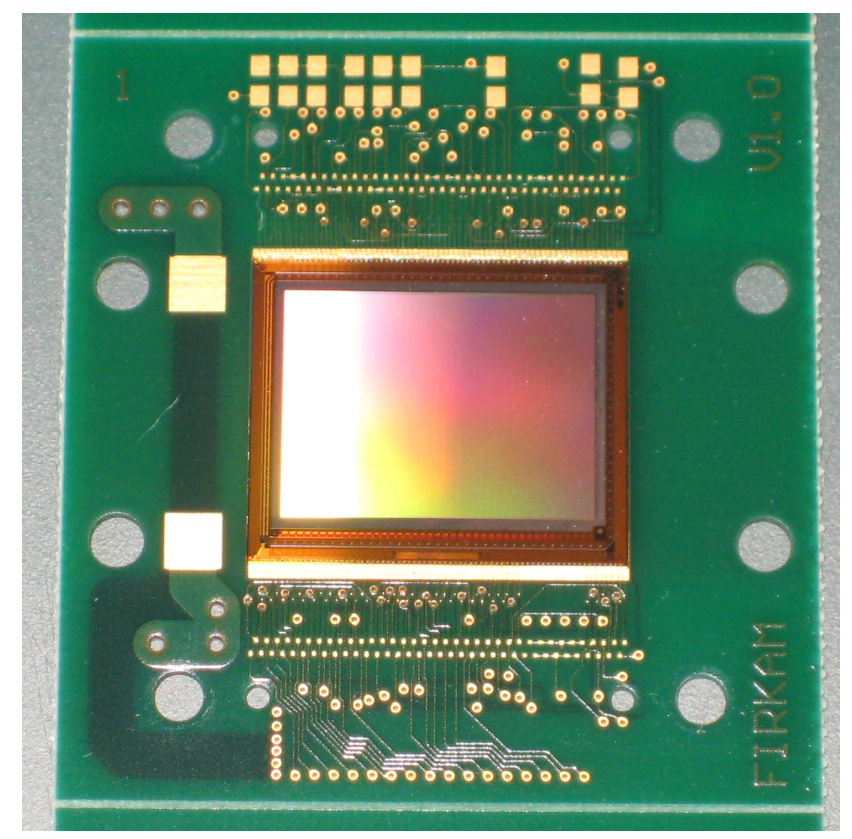

Fig. 6. Photo of the VGA-IRFPA bonded on a PCB-board.

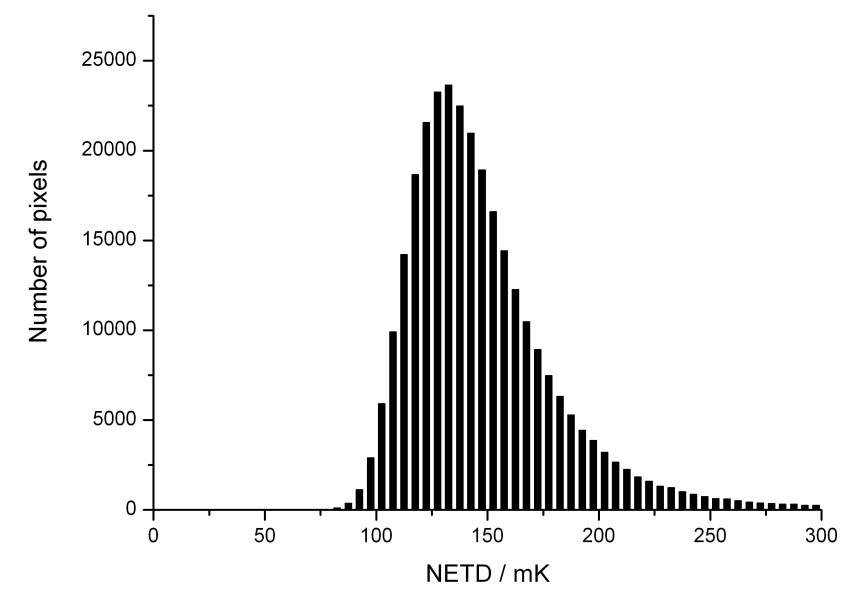

Fig. 7. NETD histogram.

SDM-readout followed by SINC-filters. The pixel-pitch of the microbolometers is $25 \mu \mathrm{m}$. First NETD measurement results are shown. In near future better results with the 2 nd generation of microbolometers are expected.

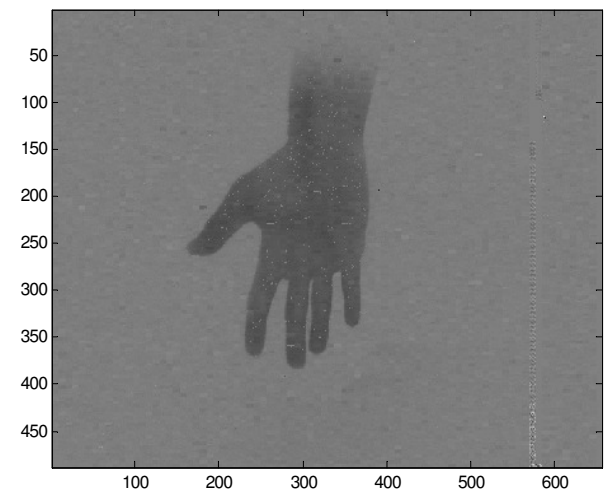

Fig. 8. Picture of a hand taken with the presented VGA-IRFPA.

Acknowledgements. The presented work is part of project "FIRKAM" funded by the German "Bundesministerium fur Bildung und Forschung BMBF". The authors would like to thank the program manager "VDI Technologiezentrum" and the FIRKAM project partners for their helpful information and discussions. We highly appreciate the support of the engineers and technicians of Fraunhofer IMS during design, fabrication, and characterization of the IRFPA.

\section{References}

Blackwell, R., Lacroix, D., Bach, T., Ishii, J., Hyland, S., Geneczko, J., Chan, S., Sujlana, B., and Joswick, M.: Uncooled $\mathrm{VO}_{\mathrm{x}}$ thermal imaging systems at BAE Systems", Proc. SPIE, 6940, 694021, 1-8, 2008.

Durand, A., Minassian, C., Tissot, J. L., Vilain, M., Robert, P., Touvignon, A., Chiappa, J. M., and Pistre, C.: Uncooled amorphous silicon TEC-less 1/4 VGA IRFPA with $25 \mu \mathrm{m}$ pixel-pitch for high volume applications, Proc. SPIE, 7298, 72982B, 1-7, 2009.

Russ, M., Bauer, J., and Vogt, H.: The geometric design of microbolometer elements for uncooled focal plane arrays, Proc. SPIE Conference Infrared Technology and Applications XXXIII, 6542, 1-10, 2007.

Weiler, D., Russ, M., Würfel, D., Lerch, R., Yang, P., Bauer, J., and Vogt, H.: A digital $25 \mu \mathrm{m}$ pixel-pitch uncooled amorphous silicon TEC-less VGA-IRFPA with massive parallel Sigma-DeltaADC readout, Proc. of SPIE, 7660, 76600S-1-7, 2010.

Norsworthy, S. R., Schreier, R., and Temes, G. C.: Delta-Sigma Data Converters, IEEE Press, 0-7803-1045-4, 1997. 